\title{
Solution-Processable Porous Nanoparticles of a Conjugated \\ Ladder Polymer Network
}

Alexander J. Kalin ${ }^{1}$, Sai Che ${ }^{1}$, Chenxu Wang ${ }^{2}$, Anthony U. Mu ${ }^{1}$, E. Meir Duka ${ }^{1}$ and Lei Fang ${ }^{1,2 *}$

${ }^{1}$ Department of Chemistry, Texas A\&M University, 3255 TAMU, College Station, TX 77843-

3255, USA

${ }^{2}$ Department of Materials Science \& Engineering, Texas A\&M University, 3003 TAMU, College

Station, TX 77843-3003, USA

Email: fang@chem.tamu.edu

1. General Information ............................................................................................ S2

2. Synthesis........................................................................................................ S3

3. ${ }^{13} \mathrm{C}$ Nuclear Magnetic Resonance ...................................................................... S5

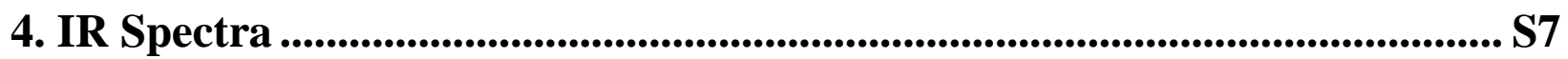

5. Dynamic Light Scattering of Nanoparticle Suspensions................................... S9

6. Optical Spectra ...................................................................................................... S12

7. $\mathrm{N}_{2}$ sorption data...................................................................................................... S14

8. Thermogravimetric Analysis ........................................................................... S16

9. References ................................................................................................................... S17 


\section{General Information}

Nanomaterials can pose significant health and safety hazards, and appropriate exposure precautions should be taken. Monomers M1 M4 were synthesized according to literature reports. ${ }^{1,2}$ Monomers M1 and M3 were purified prior to polymerization by preparative size-exclusion chromatography on a JAI recycling HPLC with SEC columns; M2 and M4 were purified by recrystallization. All other reagents were used as purchased. Toluene was dried by an Inert Technology PureSolv-MD-5a solvent purification system. Miniemulsions were generated with a Heischler UP50H probe sonicator. Field-emission scanning electron microscopic (SEM) images were collected using a FEI Quanta 600 FE-SEM at $20 \mathrm{kV}$. Samples were sputter coated in gold prior to imaging. Spin-coating of thin films was performed using an SCS spin coater from $\mathrm{CHCl}_{3}$ dispersions at $0.5 \mathrm{mg} / \mathrm{mL}$ of the nanoparticles onto octadecyltrichlorosilane (OTS)-coated $\mathrm{SiO}_{2} / \mathrm{Si}$ wafers. 


\section{Synthesis}
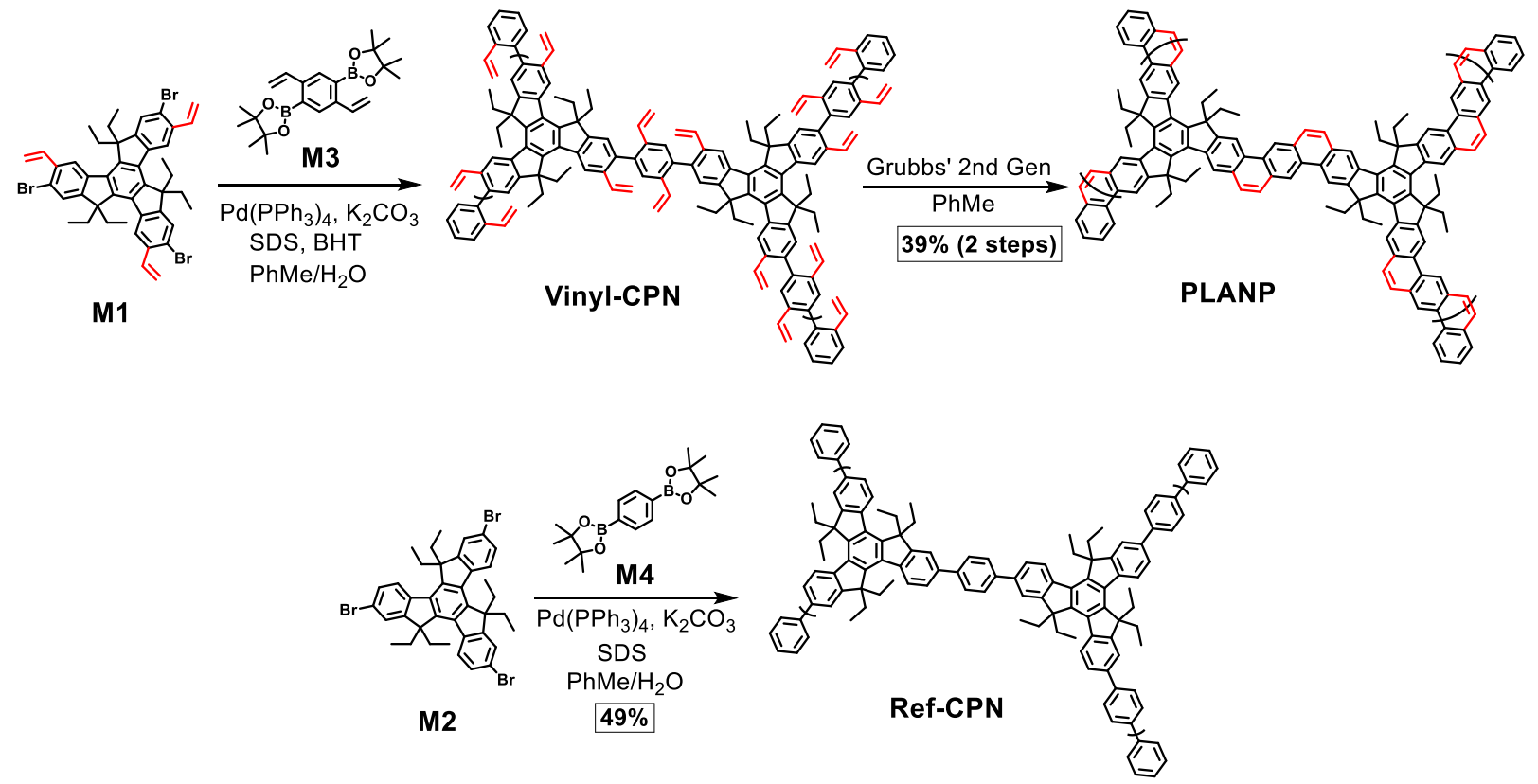

Scheme S1. Polymerization and annulation conditions for Vinyl-CPN, PLANP, and Ref-CPN.

Vinyl-CPN (Miniemulsion Polymerization): DI water $(50 \mathrm{~mL})$ was added to a two-necked round bottom flask and degassed by bubbling $\mathrm{N}_{2}$. Sodium dodecyl sulfate $(2.178 \mathrm{~g}, 150 \mathrm{mM})$ was added under $\mathrm{N}_{2}$ and stirred until dissolved. M1 (0.222 g, $0.269 \mathrm{mmol}, 2 \mathrm{eq}), \mathbf{M 3}(0.154 \mathrm{~g}, 0.403 \mathrm{mmol}, 3 \mathrm{eq}), \mathrm{Pd}\left(\mathrm{PPh}_{3}\right)_{4}(0.032$ $\mathrm{g}, 0.027 \mathrm{mmol})$, and BHT (60 mg) were dissolved in degassed, dry toluene $(5 \mathrm{~mL})$ and further degassed by three cycles of freeze-pump-thaw. The organic phase was injected into the aqueous surfactant solution under a high rate of stirring. Keeping the flask under nitrogen, a Heischler UP50 probe sonicator was used to sonicate the mixture for 10 minutes, until a homogeneous miniemulsion was formed. $\mathrm{K}_{2} \mathrm{CO}_{3}(0.379 \mathrm{~g}$, $2.74 \mathrm{mmol}, 20 \mathrm{eq})$ was added to the emulsion, which was resealed and heated to $80{ }^{\circ} \mathrm{C}$ for $24 \mathrm{~h}$. Subsequently, 2-bromostyrene $(0.1 \mathrm{~mL})$ as an end-capping agent was added and allowing to react for an additional $6 \mathrm{~h}$, before repeating the end-capping process with 2-vinylphenylboronic acid pinacol ester $(0.2$ $\mathrm{mL}$ ). After cooling down to room temperature, Amberlyst IRA-900 was added and stirred for $2 \mathrm{~h}$. The resin beads were filtered out and the resulting biphasic mixture was transferred to a separatory funnel where the organic products were extracted with $\mathrm{CH}_{2} \mathrm{Cl}_{2}$. The combined organic solution was dried over $\mathrm{MgSO}_{4}$ before 
the solvent was removed by rotary evaporation, to give the Vinyl-CPN product ( $355 \mathrm{mg}, 0.228 \mathrm{mmol}, 85 \%$ crude yield). The product was used for RCM without further purification. Solid-state ${ }^{13} \mathrm{C}\left\{{ }^{1} \mathrm{H}\right\}$ NMR: $\delta$ 151.11, 142.96, 138.17, 135.03, 126.32, 111.22, 55.90, 28.45, 6.85. FTIR $\left(\mathrm{cm}^{-1}\right): 3070.68,2962.66$, 2918.30, 2873.94, 2848.86, 1471.69, 987.55, 893.04, 748.38, 692.44.

PLANP (Ring-Closing Metathesis): Vinyl-CPN (355 mg, $0.228 \mathrm{mmol}$ ) and Grubbs' $2^{\text {nd }}$ Generation catalyst $(0.015 \mathrm{~g}, 0.018 \mathrm{mmol})$ were dispersed in toluene $(5 \mathrm{~mL})$ and heated to $100{ }^{\circ} \mathrm{C}$ in a flask shielded from light. An additional portion of Grubbs $2^{\text {nd }}$ generation catalyst $(0.045 \mathrm{~g}, 0.053 \mathrm{mmol})$ was dissolved in toluene $(10 \mathrm{~mL})$ and added via syringe pump over $3 \mathrm{hr}$. The reaction was stirred overnight, then cooled and concentrated by rotary evaporation. The product was precipitated into methanol followed by filtration using a $0.5 \mu \mathrm{m}$ membrane filter to give PLANP as a dark solid (139 mg, $0.100 \mathrm{mmol}, 39 \%$ two-step yield). Solidstate ${ }^{13} \mathrm{C}\left\{{ }^{1} \mathrm{H}\right\}$ NMR: $\delta 151.72,145.06,139.60,131.07,126.73,115.61,57.64,30.19,7.90$. FTIR $\left(\mathrm{cm}^{-1}\right)$ : 2962.66, 292.01, 2873.94, 2848.86, 1456.26, 1377.17, 887.26, 794.67, 746.45, 692.44.

Ref-CPN: Through the same miniemulsion polymerization described above using M2 (0.209 g, 0.280 mmol) and M4 (0.139 g, $0.421 \mathrm{mmol})$, Ref-CPN was isolated as a pale solid (172 $\mathrm{mg}, 0.138 \mathrm{mmol}, 49 \%)$. Solid-state ${ }^{13} \mathrm{C}\left\{{ }^{1} \mathrm{H}\right\}$ NMR: $\delta 152.25,138.46,125.58,55.70,28.43,6.33$. FTIR $\left(\mathrm{cm}^{-1}\right): 3030.17,2962.66$, 2929.87, 2872.01, 1473.62, 1458.18, 1375.25, 887.26, 831.32, 806.25, 758.02, 696.30.

Polystyrene:PLANP dispersion for composite films: To form the PS:PLANP dispersions, polystyrene $\left(M_{\mathrm{n}} 192 \mathrm{~kg} \mathrm{~mol}{ }^{-1} ; 60 \mathrm{mg}\right)$ was dissolved in $\mathrm{CHCl}_{3}(10 \mathrm{~g})$. To this solution, PLANP (5 wt\% of PS) was added and sonicated to homogenize to form the final dispersion. From these dispersions, films could be formed by drop casting onto a glass slide, or by evaporation from a vial. The films were left to dry overnight and then delaminated from the glass surface for measurements. 


\section{3. ${ }^{13} \mathrm{C}$ Nuclear Magnetic Resonance}

Solid-state proton-decoupled ${ }^{13} \mathrm{C}$ NMR spectra were obtained on a Bruker Avance $400 \mathrm{MHz}$ spectrometer with $4 \mathrm{~mm} \mathrm{CP} / \mathrm{MAS}$ probes and MAS rates of $10 \mathrm{kHz}$ at room temperature. Peaks were assigned in accordance with literature. ${ }^{2}$

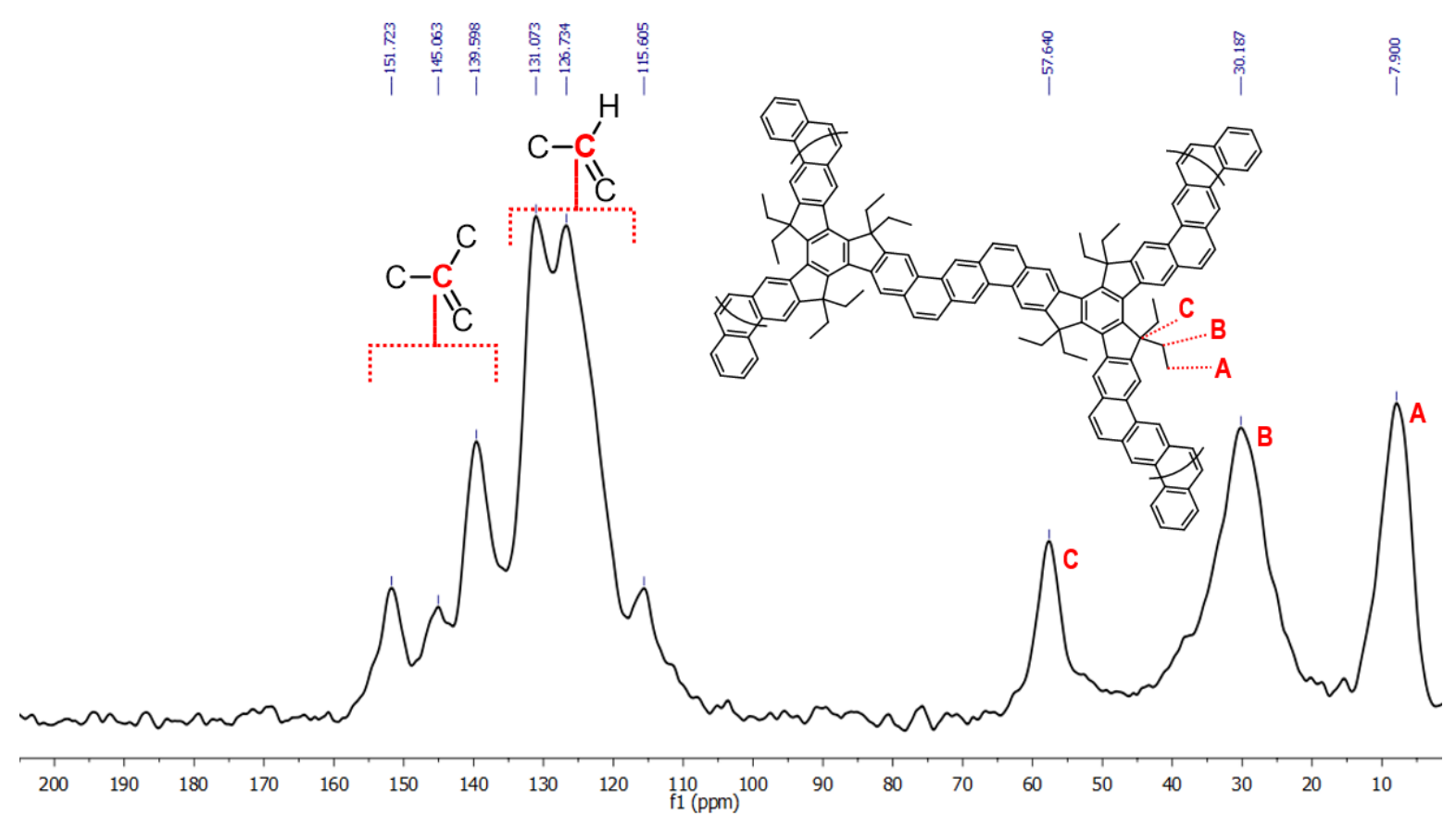

Figure S1. ${ }^{13} \mathrm{C}$ CP/MAS NMR Spectra of PLANP 


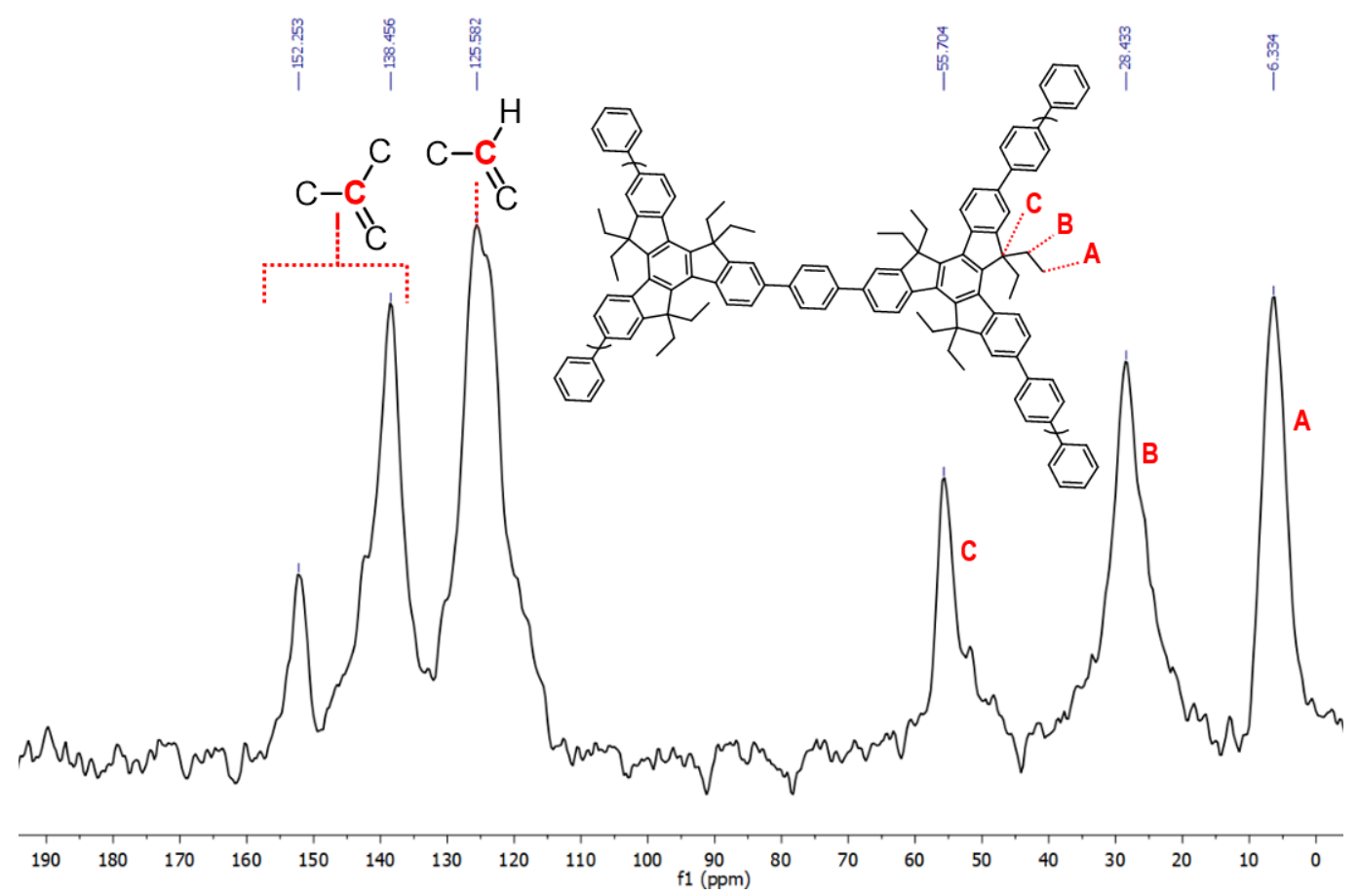

Figure S2. ${ }^{13} \mathrm{C}$ CP/MAS NMR Spectra of Ref-CPN

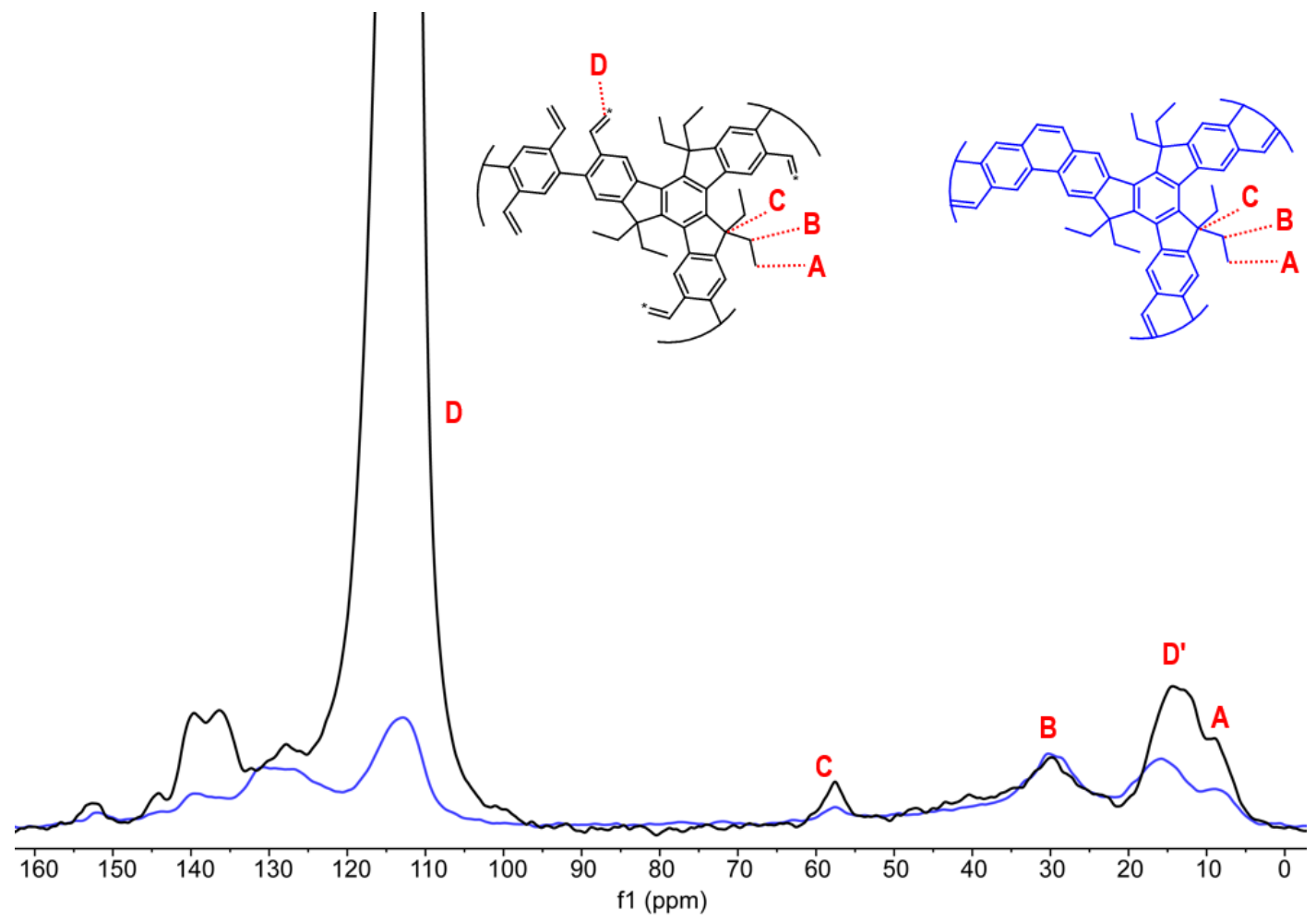

Figure S3. ${ }^{13} \mathrm{C}$ CP/MAS NMR spectra of ${ }^{13} \mathrm{C}$-labeled Vinyl-CPN (labeled carbon marked with asterisk) and spectra of PLANP after RCM. D' is the side band of the ${ }^{13} \mathrm{C}$ isotope labeled carbon. 


\section{IR Spectra}

Fourier transform infrared spectroscopy (FT-IR) spectra were recorded by ZnSe attenuated total reflection with a Shimadzu IRAffinity-1S spectrometer. Powders of the samples were tested with no prior preparation. All nanoparticles showed $\mathrm{sp}^{3}$ (moderate intensity) and $\mathrm{sp}^{2}$ (weak to negligible) $\mathrm{C}-\mathrm{H}$ stretches at $2900-3100 \mathrm{~cm}^{-1}$, aromatic $\mathrm{C}=\mathrm{C}$ stretching around $1500 \mathrm{~cm}^{-1}$, and $\mathrm{C}-\mathrm{H}$ bending peaks between $650-950$ $\mathrm{cm}^{-1}$. As aromatic $\mathrm{C}-\mathrm{H}$ bends were used for structural comparisons, small molecular precursors for fingerprint region comparisons were chosen to compare with that of the nanoparticles.

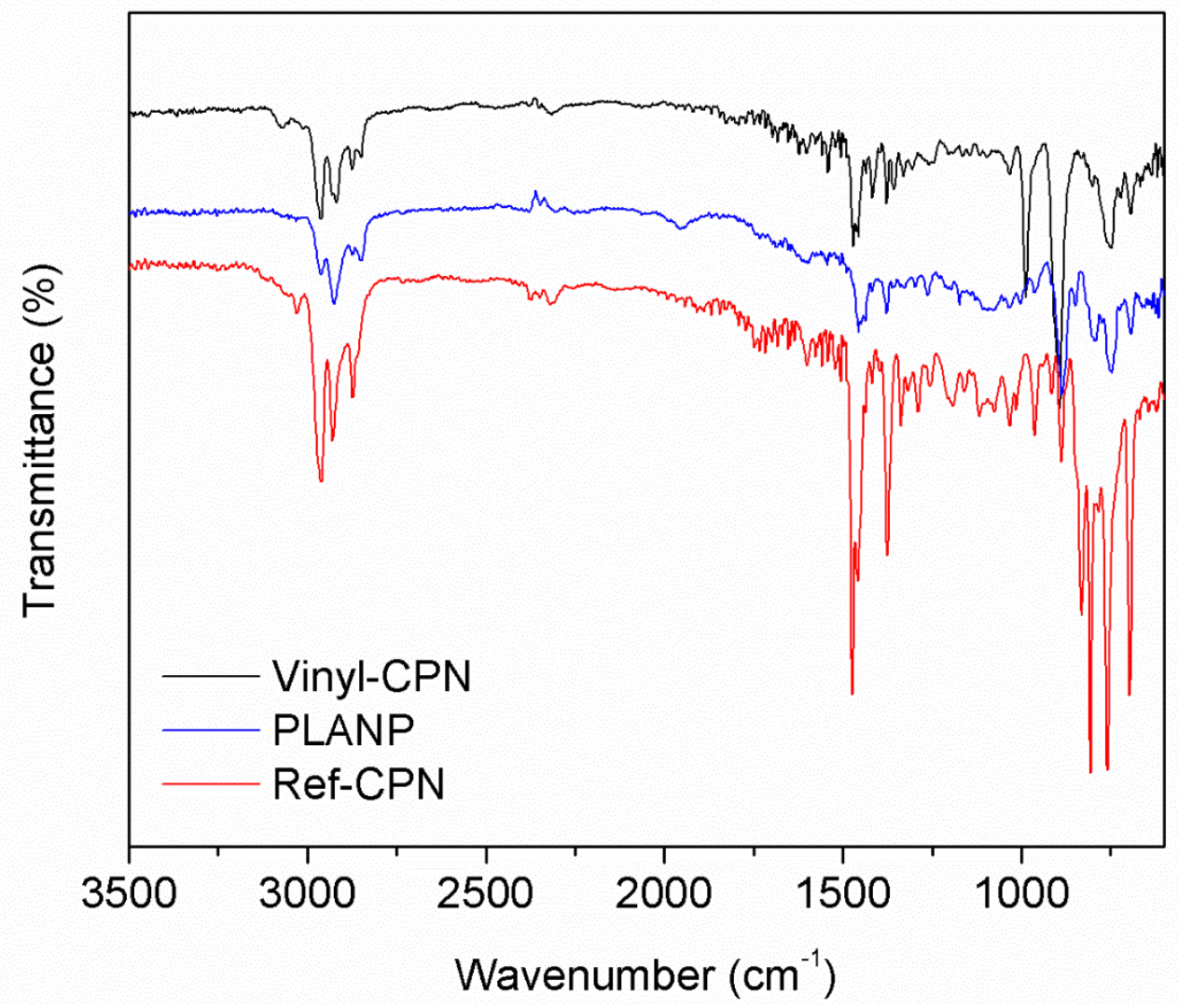

Figure S4. FTIR Spectra of Vinyl-CPN, PLANP, and Ref-CPN. 


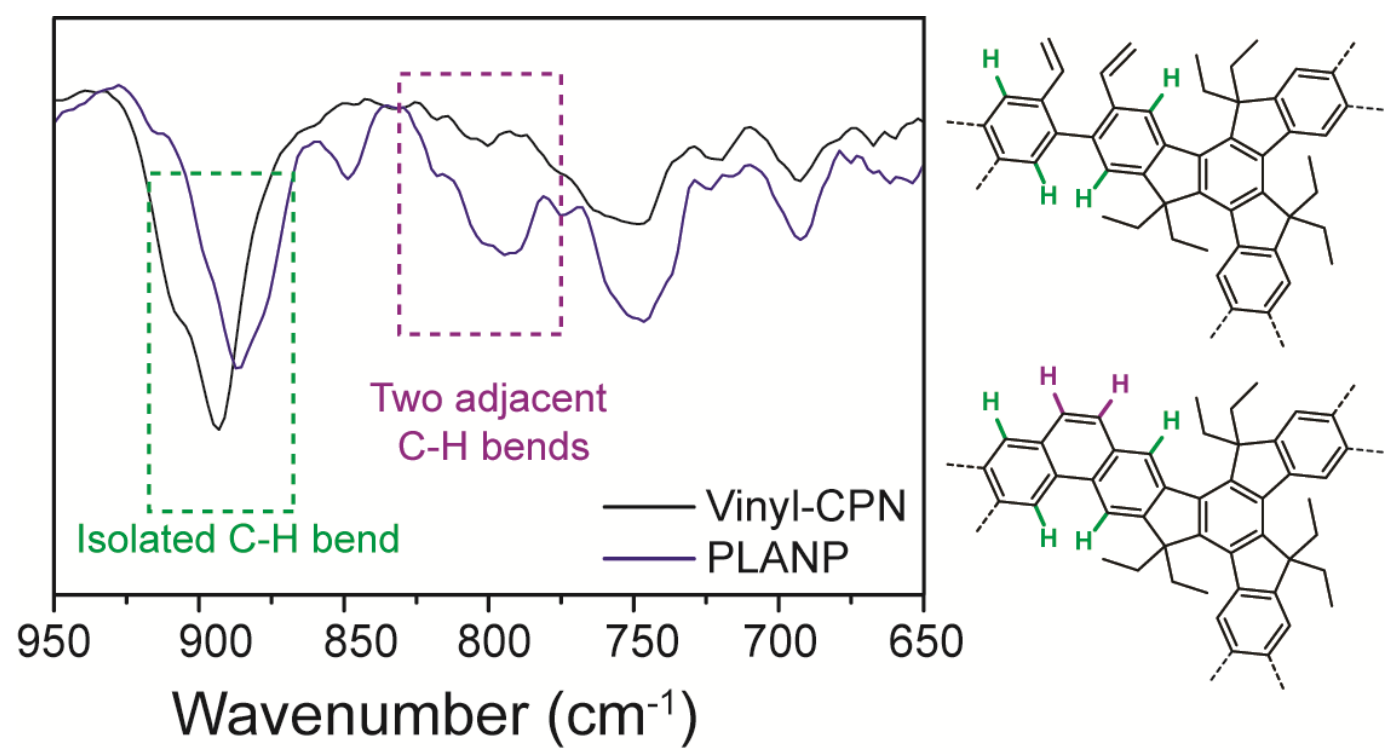

Figure S5. FTIR fingerprint region of PLANP and Ref-CPN, with peaks highlighted according to corresponding structures.

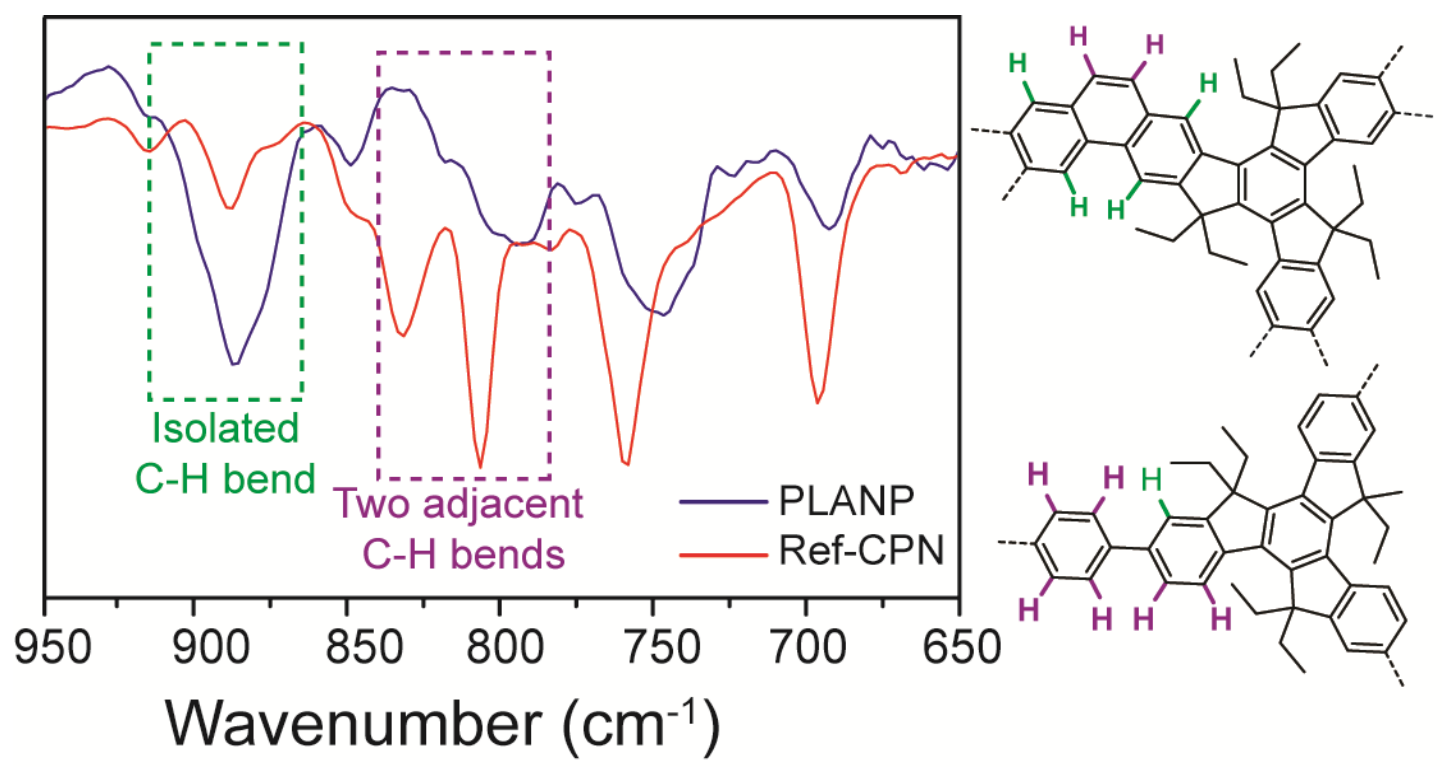

Figure S6. FTIR fingerprint region of PLANP and Ref-CPN, with peaks highlighted according to corresponding structures. 


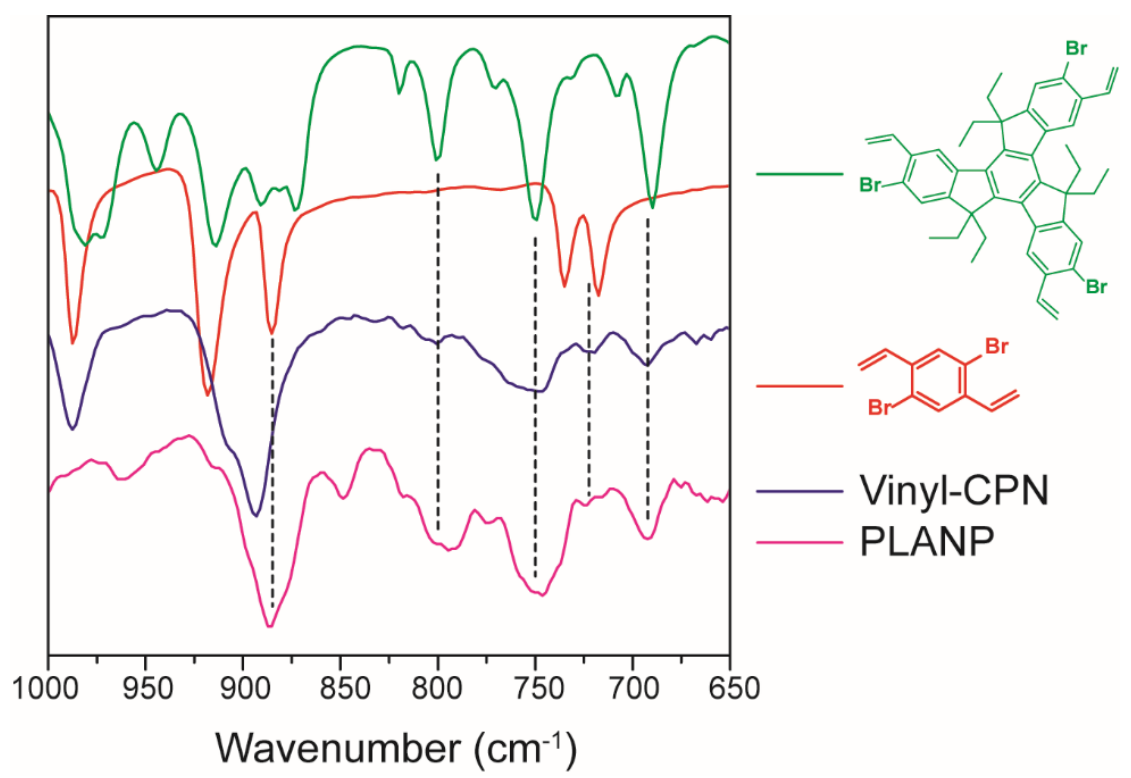

Figure S7. FTIR fingerprint region of PLANP, Vinyl-CPN, and truxene and phenylene precursors.

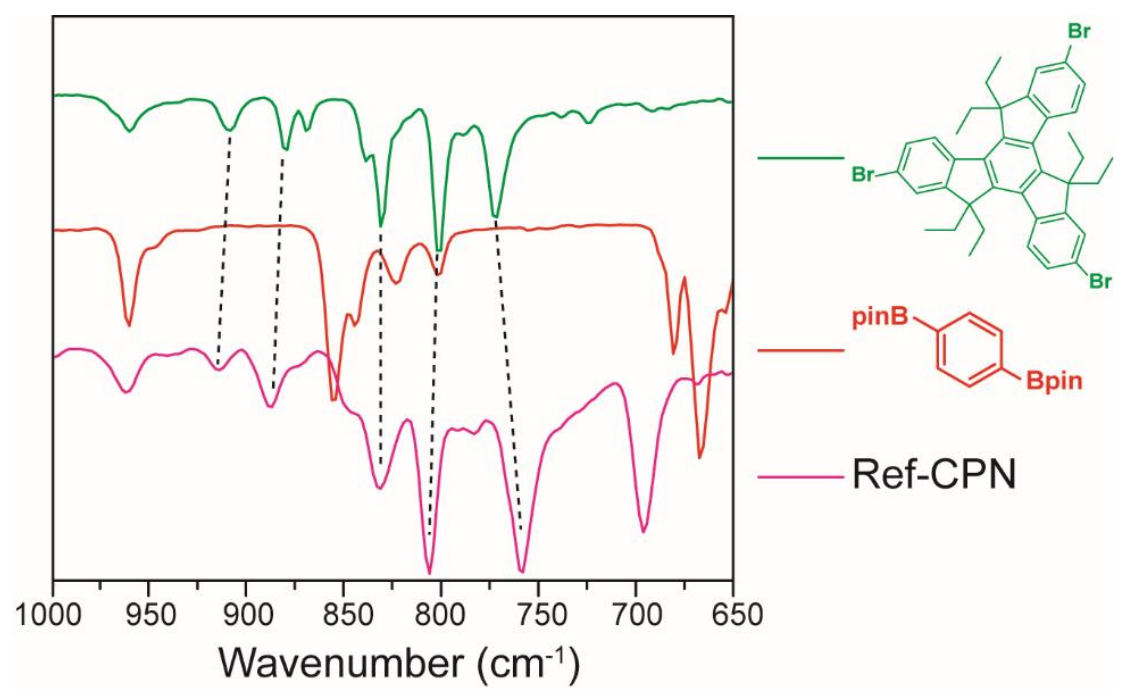

Figure S8. FTIR fingerprint region of PLANP, Vinyl-CPN, and truxene and phenylene precursors.

\section{Dynamic Light Scattering of Nanoparticle Suspensions}

DLS data of nanoparticle dispersions was obtained on a Malvern Zetasizer Nano ZS. Nanoparticles were dispersed into $\mathrm{CHCl}_{3}$ at approximately $0.5 \mathrm{mg} / \mathrm{mL}$ and measured in a quartz cuvette. Data from three measurements runs for each sample were averaged together by the Malvern software package, giving the data below and the values in Table 1. 


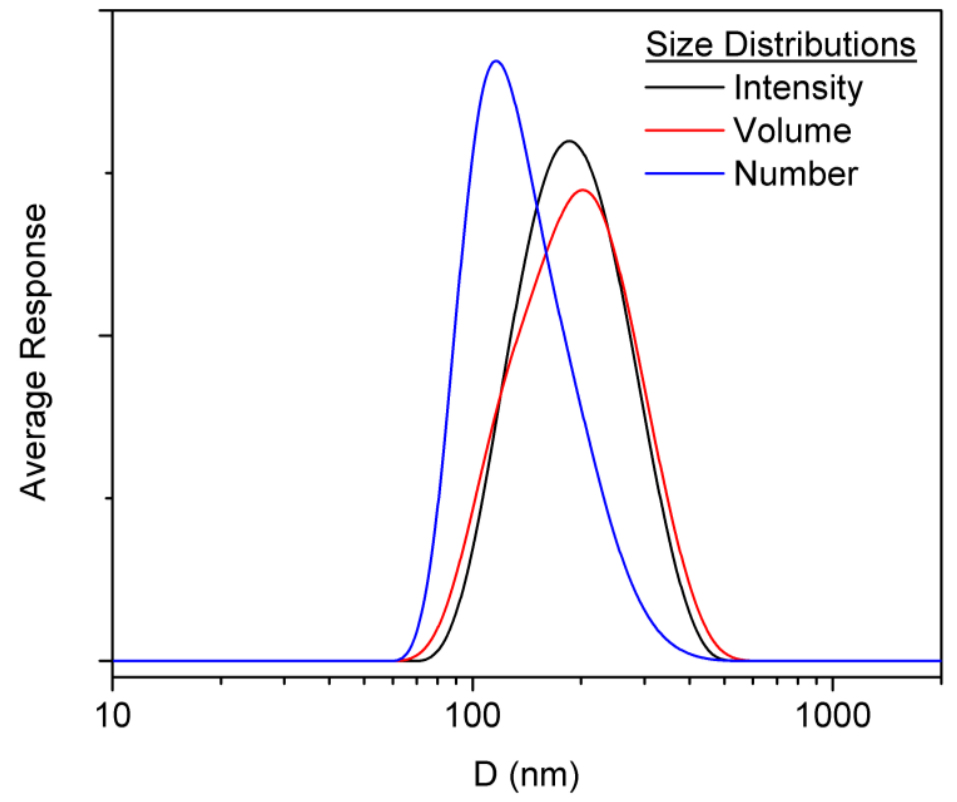

Figure S9. DLS size distributions of PLANP.

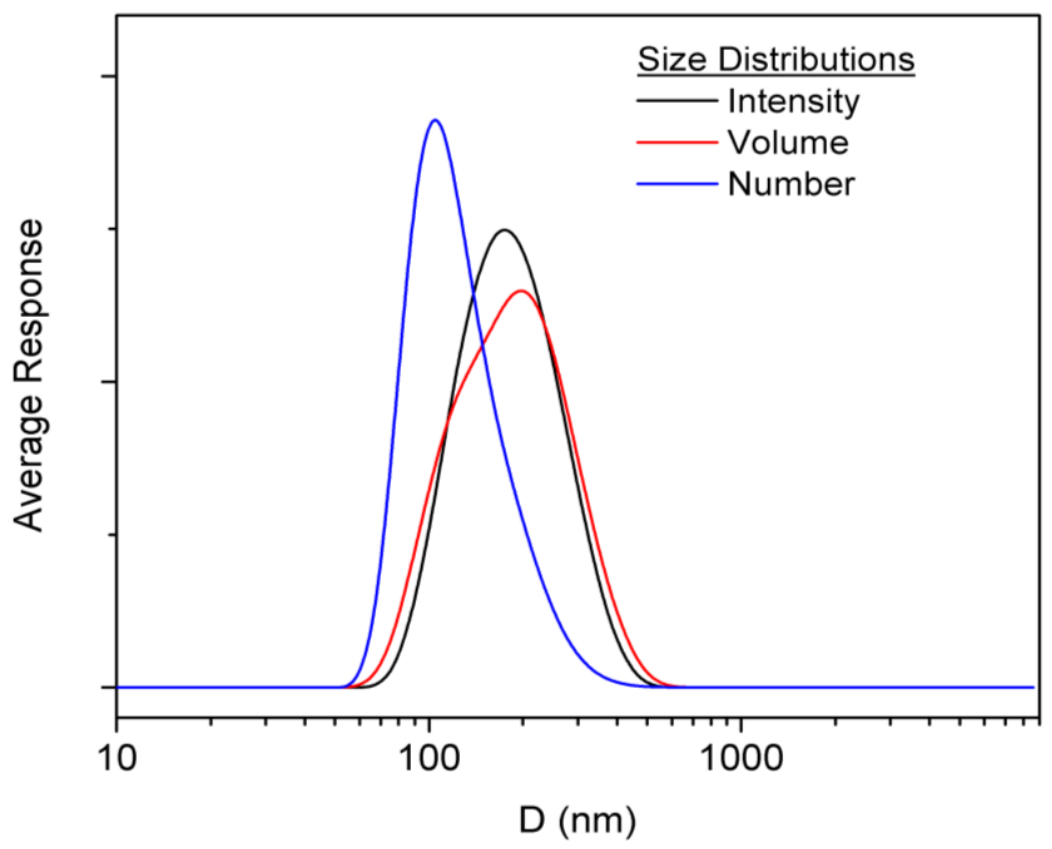

Figure S10. DLS size distributions of Ref-CPN. 


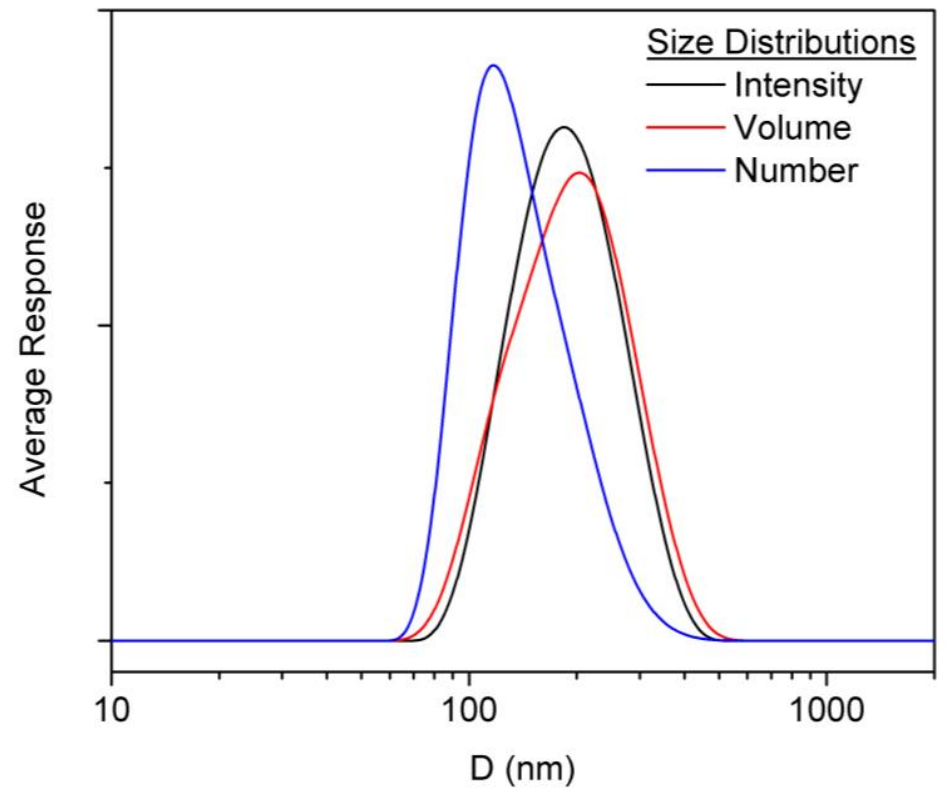

Figure S11. DLS size distributions of Vinyl-CPN. 


\section{Optical Spectra}

UV-Visible absorption spectroscopy was performed on a Shimadzu UV-2600 and fluorescence emissions were measured on a Horiba Fluoromax-4. For solution-phase spectra, nanoparticles were dispersed in $\mathrm{CHCl}_{3}$ at low concentrations $(0.5-1.5 \mathrm{mg} / \mathrm{L})$ to prevent detector saturation and measured in quartz cuvettes. Emission measurements were performed with $5 \mathrm{~nm}$ slit widths. Excitation wavelengths $\left(\lambda_{\mathrm{ex}}\right)$ are noted below each figure.

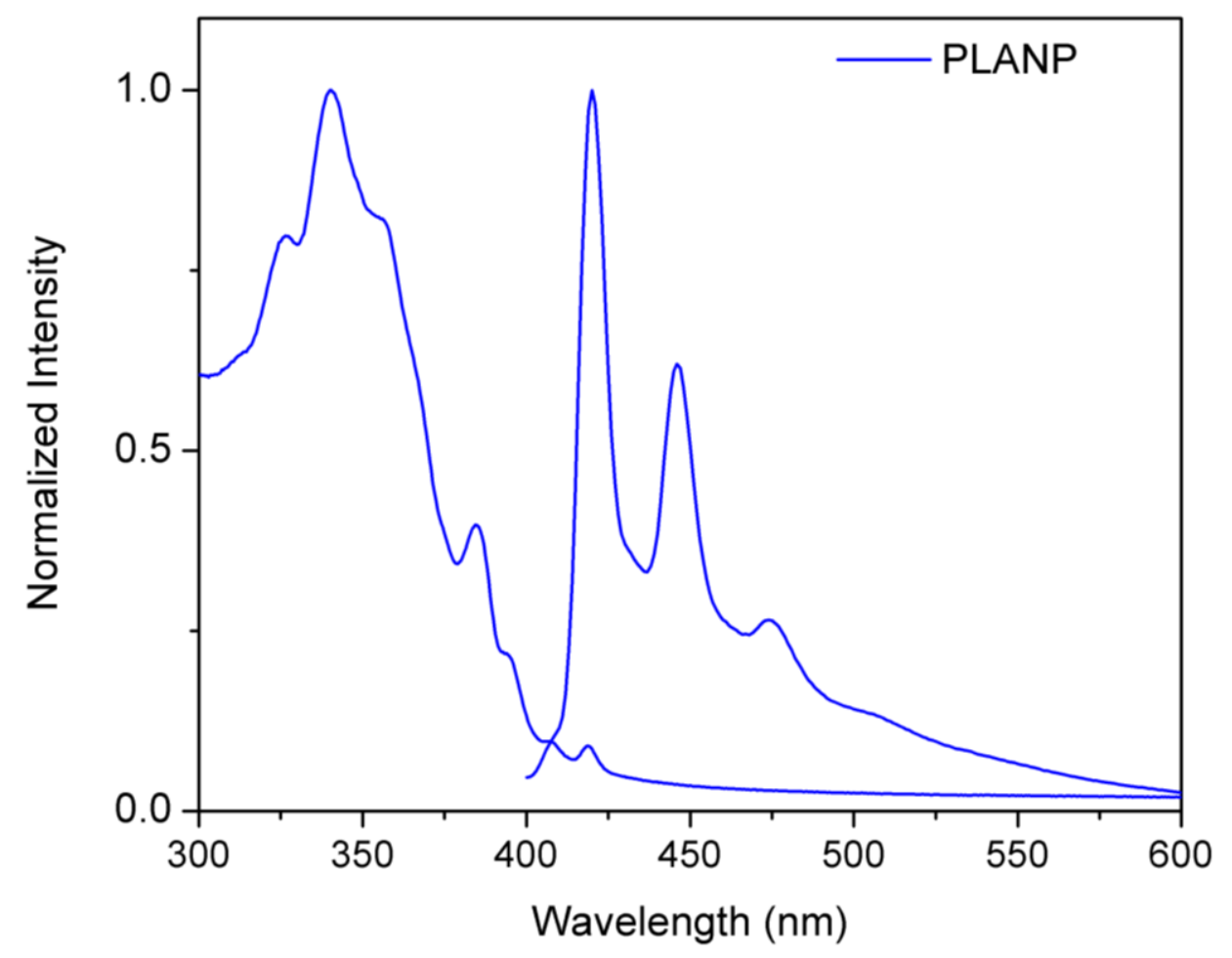

Figure S12. UV-Vis absorbance and emission $\left(\lambda_{\mathrm{ex}}=385 \mathrm{~nm}\right)$ spectra of PLANP. The Stokes shift between the weak lowest energy absorbance peak and the highest energy emission peak is $<1 \mathrm{~nm}$. 


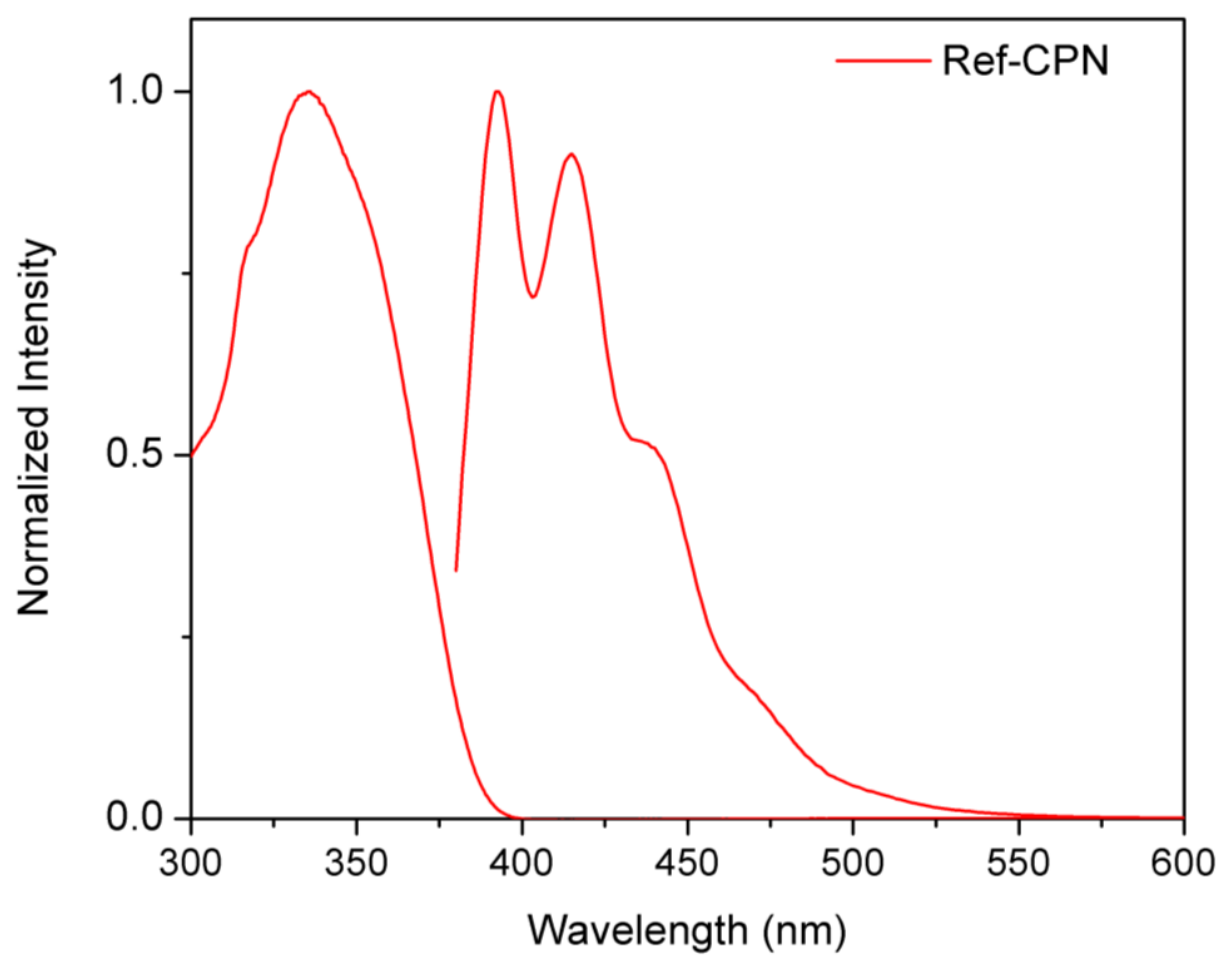

Figure S13. UV-Vis absorbance and emission $\left(\lambda_{\mathrm{ex}}=365 \mathrm{~nm}\right)$ spectra of Ref-CPN. Ref-CPN possesses a slightly lower $\lambda_{\max }$ than PLANP, with a Stokes shift of $57 \mathrm{~nm}$.

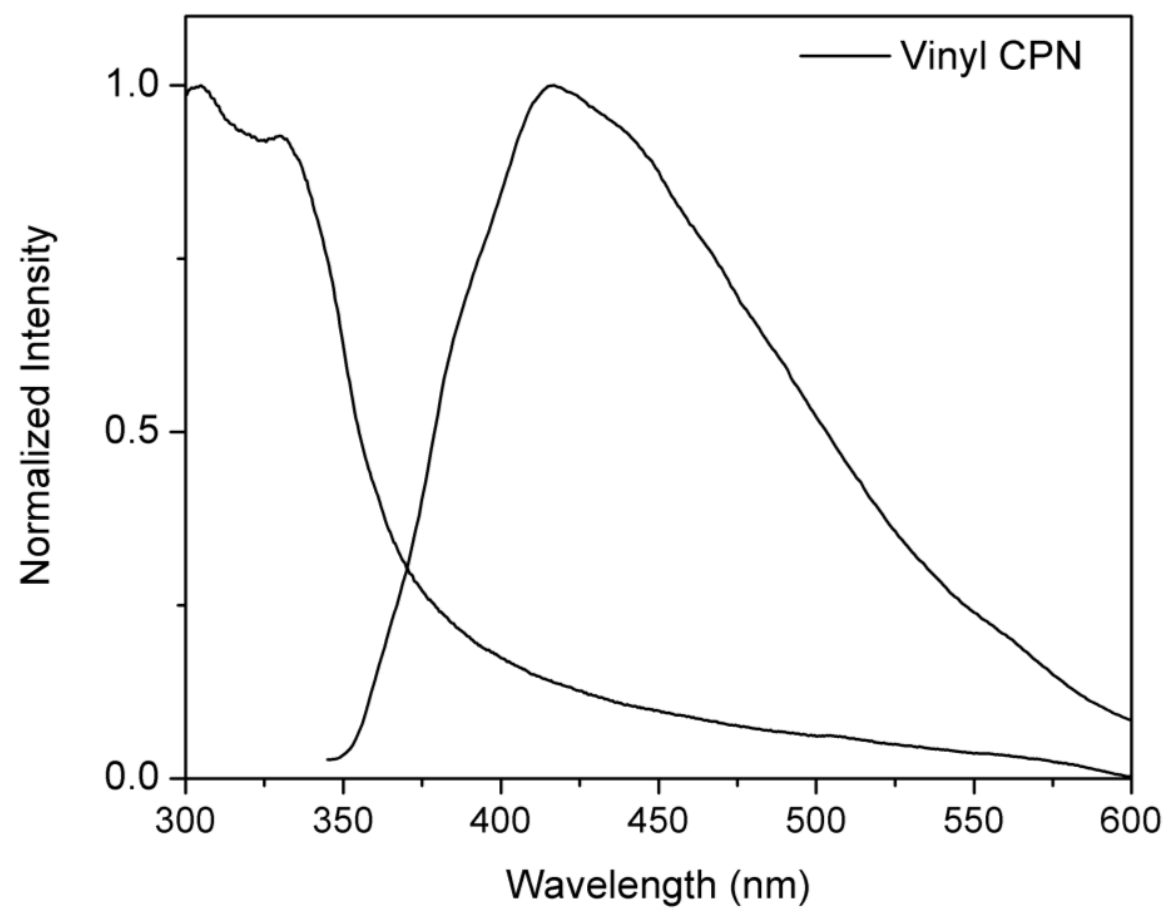

Figure S14. UV-Vis absorbance and emission $\left(\lambda_{\mathrm{ex}}=350 \mathrm{~nm}\right)$ spectra of Vinyl-CPN. Similar to Ref-CPN, Vinyl-CPN exhibits broad spectra, with a Stokes shift of $87 \mathrm{~nm}$. 


\section{7. $\mathrm{N}_{2}$ sorption data}

$\mathrm{N}_{2}$ sorption measurements were performed on a Micromeritics ASAP 2020 surface area and pore size analyzer. Prior to the gas adsorption measurements, the sample was degassed for $10 \mathrm{~h}$ at $120{ }^{\circ} \mathrm{C}$. Pore size distribution data were calculated from the $\mathrm{N}_{2}$ sorption isotherms based on the Nonlocal Density Functional Theory (NLDFT) method in the Micromeritics ASAP 2020 software package (assuming slit pore geometry).

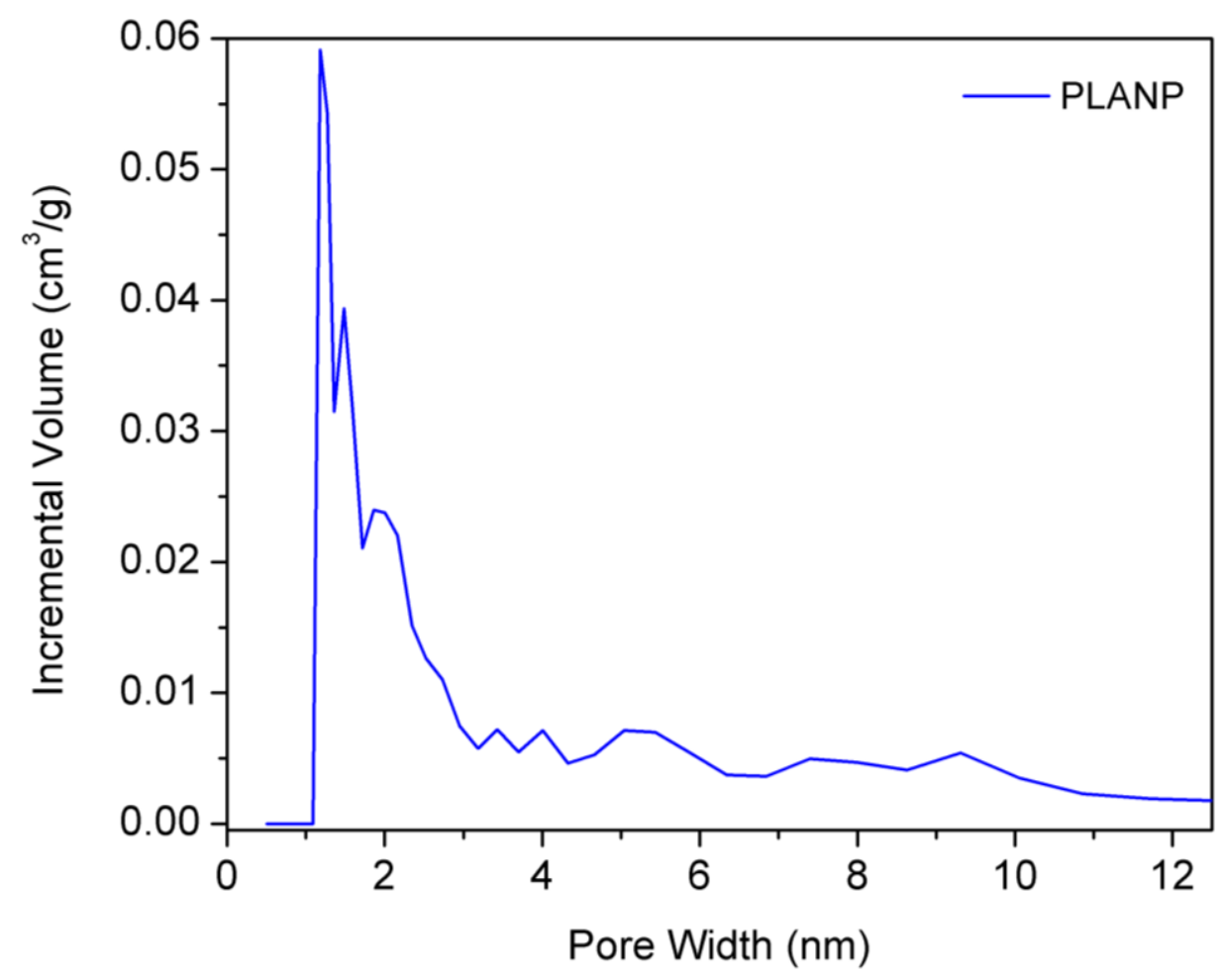

Figure S15. Pore size distribution of PLANP. 


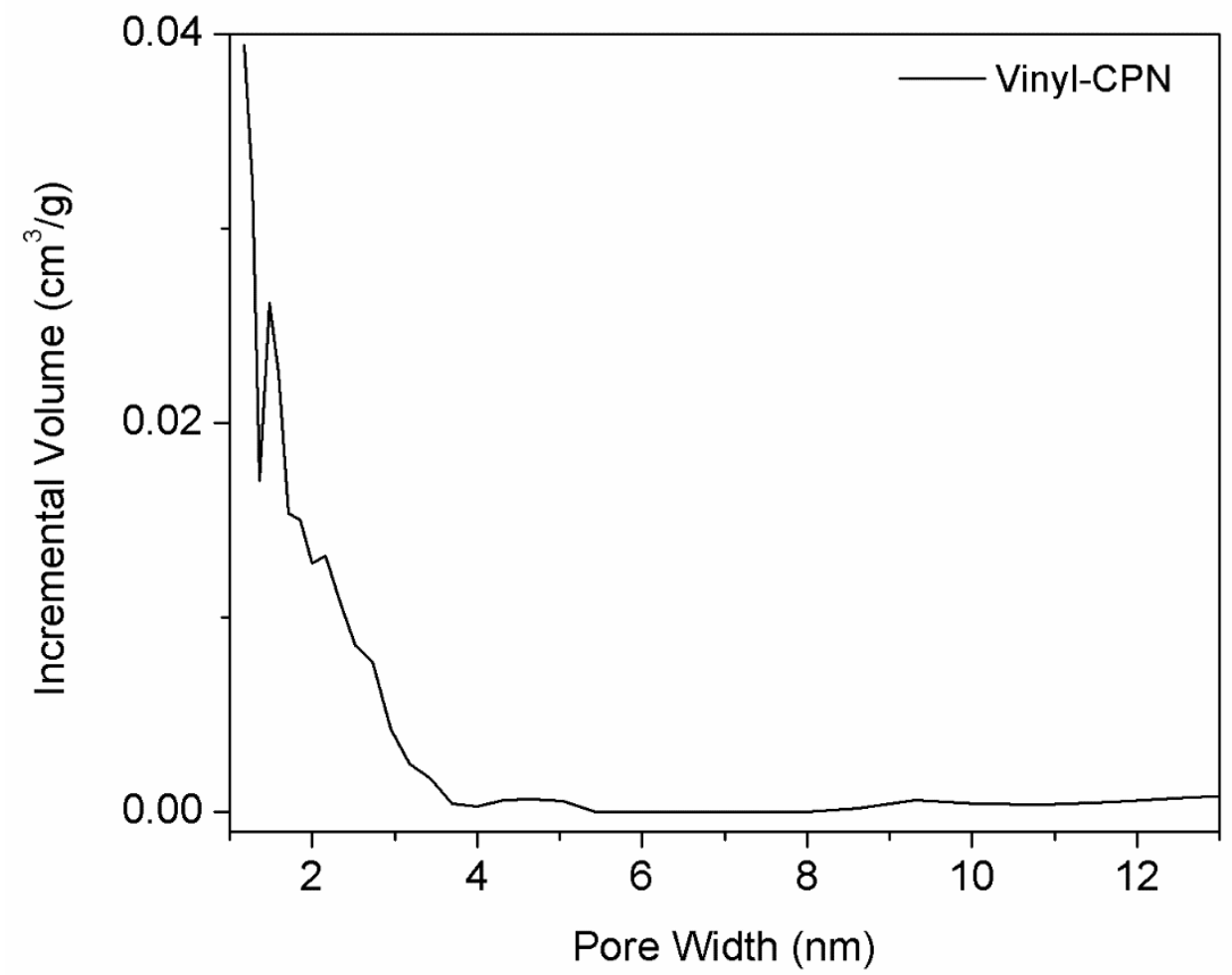

Figure S16. Pore size distribution of Vinyl-CPN. 


\section{Thermogravimetric Analysis}

Thermogravimetric analysis (TGA) data were collected on Mettler-Toledo TGA-DSC-1 at a heating rate of $20{ }^{\circ} \mathrm{C} \min ^{-1}$ from $30{ }^{\circ} \mathrm{C}$ to $900{ }^{\circ} \mathrm{C}$ under an $\mathrm{N}_{2}$ atmosphere. Decomposition temperature was calculated as the temperature at which $5 \%$ mass loss occurred.

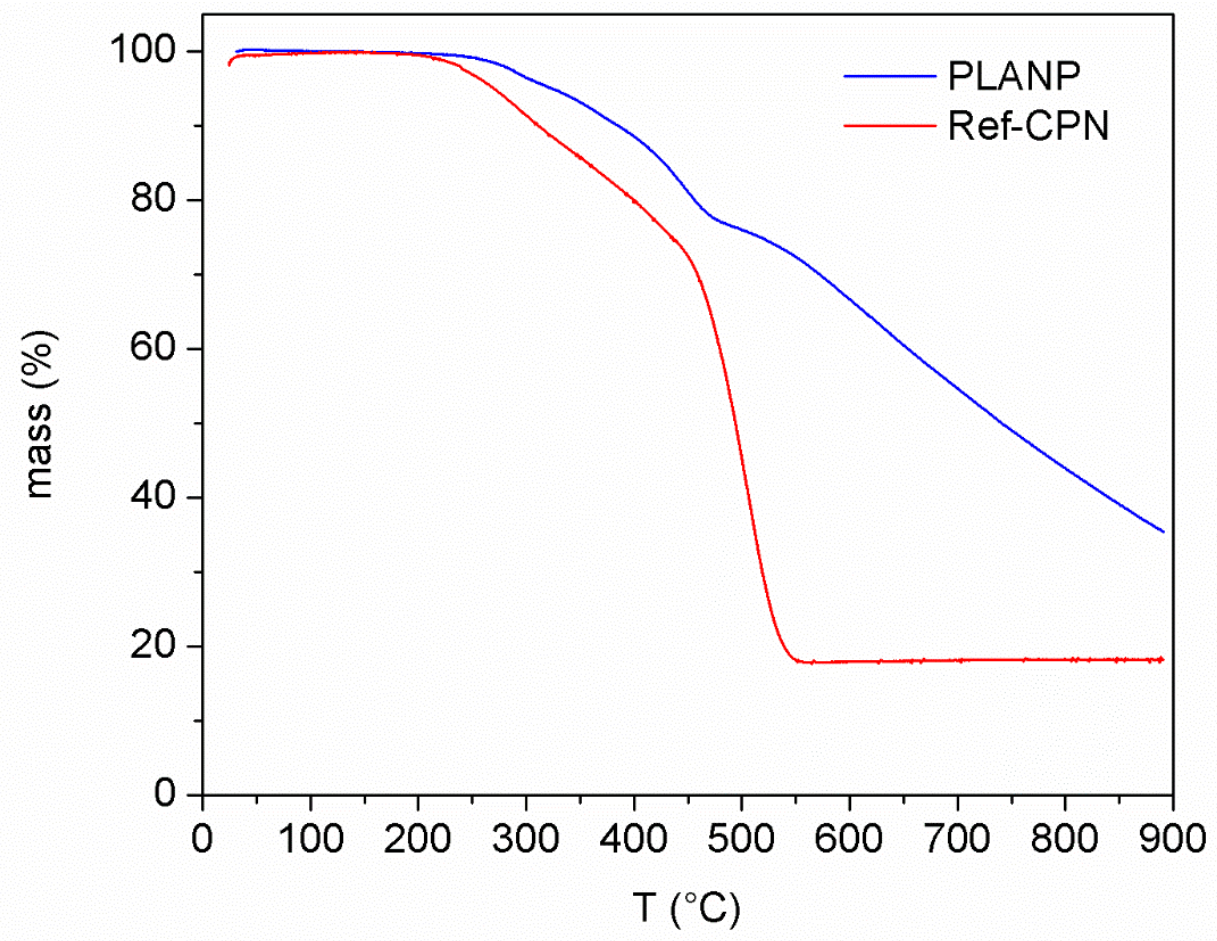

Figure S17. TGA traces of PLANP and Ref-CPN. 


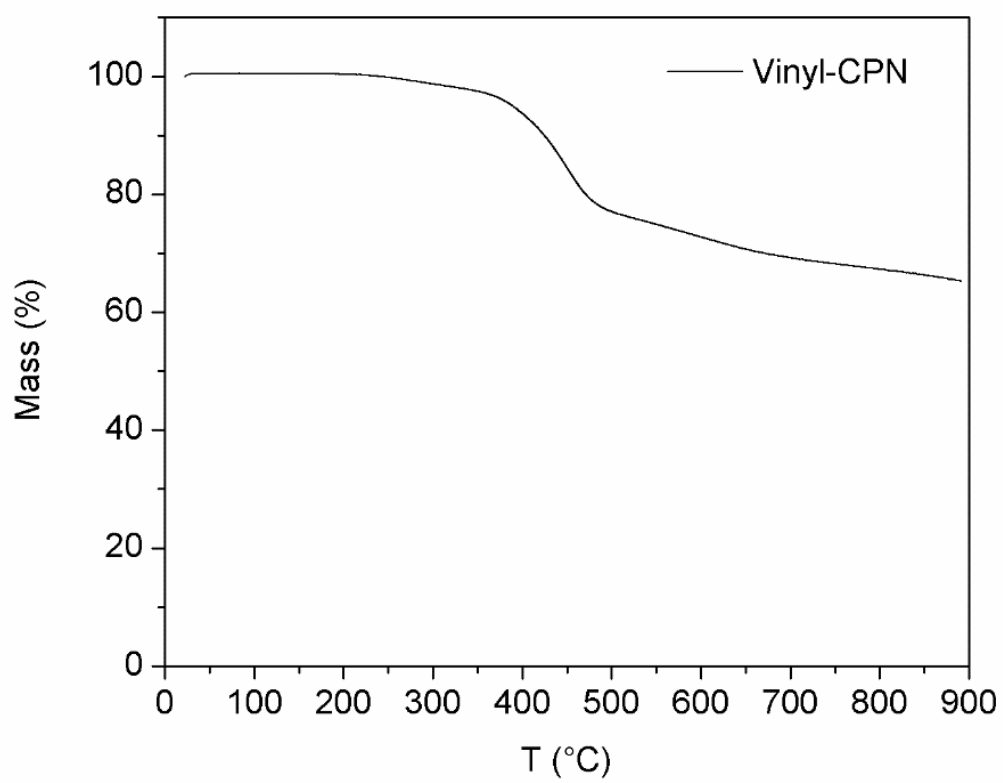

Figure S18. TGA trace of Vinyl-CPN.

\section{References}

1) Lee, J.; Li, H.; Kalin, A. J.; Yuan, T.; Wang, C.; Olson, T.; Li, H.; Fang, L. Angew. Chem. Int. Ed. 2017, 56, 13727.

2) Che, S.; Pang, J.; Kalin, A. J.; Wang, C.; Ji, X.; Lee, J.; Cole, D.; Li, J.-L.; Tu, X.; Zhang, Q.; Zhou, H.-C.; Fang, L. ACS Materials Lett. 2019, 2, 49. 